\title{
Perfil metabólico de filhotes de peixe-boi da Amazônia (Trichechus inunguis) em cativeiro, alimentados com diferentes sucedâneos do leite materno
}

\author{
[Metabolic profile of captive Amazonian manatee calves (Trichechus inunguis), fed \\ different substitutes for maternal milk] \\ A.H.P. Maduro ${ }^{1}$, V.M.F. da Silva ${ }^{1}$, R.P.M. Oliveira ${ }^{2}$, P.S. Barbosa ${ }^{1}$
}

${ }^{1}$ Laboratório de Mamíferos Aquáticos/LMA - Instituto Nacional de Pesquisas da Amazônia/INPA - Manaus, AM

${ }^{2}$ Faculdade de Ciências Agrárias/FCA - Universidade Federal do Amazonas/UFAM - Manaus, AM

\begin{abstract}
RESUMO
Dez filhotes de peixe-boi da Amazônia criados em cativeiro foram amamentados com diferentes dietas lácteas em duas etapas. Na primeira, com sucedâneo 1 (S1), utilizado rotineiramente no Inpa e, na segunda, com sucedâneo 2 (S2), elaborado com base na composição centesimal do leite materno da espécie. Cada etapa durou 62 dias, realizando-se coleta de sangue ao final de cada uma para avaliação dos valores de proteínas totais (PT), albumina (ALB), globulinas (GLO), glicose (GLI), lipídeos totais (LT), colesterol (COL), triglicerídeos (TRI), cálcio $(\mathrm{Ca})$, fósforo $(\mathrm{P})$, magnésio $(\mathrm{Mg})$, sódio $(\mathrm{Na})$, potássio $(\mathrm{K})$, hematócrito (Ht) e hemoglobina (Hb). Houve redução dos níveis séricos de PT, ALB, GLO, Ca, P, K, GLI, LT, COL e TRI, após a alimentação dos animais com S2 quando comparados aos valores obtidos com S1. Os níveis de $\mathrm{Na}$ foram maiores quando administrado o S2 $(143,47 \pm 0,99 \mathrm{mmol} / \mathrm{L})$. Valores de $\mathrm{Ht}$ e $\mathrm{Mg}$ não diferiram entre os tratamentos. O perfil metabólico, em geral, esteve dentro dos níveis normais para a espécie.
\end{abstract}

Palavras-chave: peixe-boi da Amazônia, nutrição, bioquímica sérica, hemograma

\begin{abstract}
Ten Amazonian manatee calves who live in captivity were fed with different substitutes for maternal milk in two phases. During the first phase, the calves were fed substitute 1 (S1), which is what was routinely used by INPA, and during the second phase they were fed substitute 2 (S2), which was formulated based on the nutritional composition of T. inunguis maternal milk. Each phase lasted 62 days, when blood samples were collected for analysis of values of total protein (PT), albumin (ALB), globulin (GLO), glucose (GLI), total lipids (LT), cholesterol (COL), triglycerides (TRI), calcium (Ca), phosphorus (P), magnesium $(\mathrm{Mg})$, sodium ( $\mathrm{Na}$ ), potassium $(\mathrm{K})$, hematocrit $(\mathrm{Ht})$ and hemoglobin $(\mathrm{Hb})$. There was a decrease in PT, ALB, GLO, $\mathrm{Ca}, P$ and $K$ after feeding the animals with $S 2$ when compared to values obtained with S1. Sodium levels were higher when $S 2$ was administered $(148.16 \pm 1.15 \mathrm{mmol} / \mathrm{L})$ than when S1 was given $(143.47 \pm 0.99$ mmol/l). Values of $\mathrm{Ht}$ and $\mathrm{Mg}$ did not differ between treatments. The metabolic profile was within normal levels for this species.
\end{abstract}

Keywords: Amazonian manatee, nutrition, serum biochemical, hemogram

\section{INTRODUÇÃO}

O peixe-boi da Amazônia (Trichechus inunguis) é o menor dos sirênios e o único restrito à água doce. São herbívoros monogástricos e, na natureza, alimentam-se de uma variedade de plantas (Guterres-Pazin et al., 2014).
Desde 1974, o Laboratório de Mamíferos Aquáticos do Instituto Nacional de Pesquisas da Amazônia - LMA/Inpa, em Manaus, Amazonas, Brasil, vem resgatando e reabilitando filhotes de peixe-boi da Amazônia oriundos da caça ilegal e utilizando os dados biológicos coletados desses filhotes como ferramentas no monitoramento do

Recebido em 19 de março de 2019 
processo de reabilitação dos animais (Amaral et al., 2010).

O desenvolvimento de um sucedâneo do leite materno adequado para os filhotes órfãos é de extrema importância, tendo em vista que os animais recebidos nos criadouros conservacionistas chegam com graves deficiências nutricionais, necessitando de medidas emergências quanto à nutrição para o completo restabelecimento do seu organismo (D'Affonsêca Neto e Vergara-Parente, 2006).

Alguns estudos já foram realizados para se obter uma formulação láctea artificial adequada à demanda nutricional dos filhotes de $T$. inunguis em cativeiro (Best et al., 1982; Colares et al., 1987; Rodriguez-Chacón, 2001; Rosas, 1992). Essas formulações forneceram ganho de peso e crescimento variados aos animais, contribuindo para os estudos da nutrição da espécie. Contudo, ainda não foram realizados experimentos com sucedâneos do leite com base nos níveis nutricionais do leite materno para a espécie.

Visando avaliar, diagnosticar e prevenir transtornos metabólicos, bem como indicar o estado nutricional em vacas leiteiras, Payne (1970) utilizou análise de componentes hematológicos e bioquímicos específicos. Essa metodologia se difundiu e outros autores passaram a utilizá-la inclusive para diferentes espécies de mamíferos (Ferreira e Torres, 1992; González et al., 2000; Ribeiro et al., 2003 ).

A composição bioquímica do sangue reflete, de maneira confiável, o equilíbrio entre o ingresso, o egresso e a metabolização dos nutrientes nos tecidos animais. Assim, o perfil metabólico pode ser usado para monitorar a adaptação metabólica, diagnosticar desequilíbrios da homeostase de nutrientes e revelar as causas de uma doença nutricional ou metabólica (González et al., 2000).

Silva (2008) realizou um estudo com filhotes cativos de peixe-boi marinho (Trichechus manatus) e verificou variações significativas dos parâmetros bioquímicos sorológicos entre animais amamentados com fórmula láctea artificial à base de leite de soja e os amamentados pela mãe, destacando-se a interferência da dieta no perfil metabólico desses animais.
Este estudo teve como objetivo avaliar o perfil metabólico de filhotes de peixe-boi da Amazônia (Trichechus inunguis), criados em cativeiro e alimentados com diferentes sucedâneos do leite materno, para determinação da dieta mais adequada ao desenvolvimento dos animais.

\section{MATERIAL E MÉTODOS}

O estudo foi realizado com 10 filhotes de peixesbois da Amazônia lactentes (três machos e sete fêmeas), com peso médio de $65,75 \mathrm{~kg}(17-120 \mathrm{~kg})$ e idade média de 15,3 meses (2-25 meses), mantidos em cativeiro no Parque Aquático Robin C. Best do Instituto Nacional de Pesquisas da Amazônia - Inpa e aprovado pelo Comitê de Ética em Pesquisas no Uso de Animais do Inpa, registrado sob o número de protocolo 005/2013.

Os filhotes foram mantidos em piscinas de fibra de vidro com aproximadamente $7 \mathrm{~m}^{2}$ de área e capacidade para cinco mil litros de água. Os animais foram amamentados seis vezes por dia, em intervalos de duas horas. Os experimentos foram realizados em duas etapas. Na primeira etapa, os filhotes receberam a fórmula láctea utilizada rotineiramente no Parque Aquático Robin C. Best do Inpa (S1), por um período de 62 dias.

Antes da segunda etapa, os animais passaram por um período de 38 dias de adaptação aos novos ingredientes do novo sucedâneo (S2), respeitando-se o tempo necessário para adaptação dos filhotes à nova dieta, incrementando-se os ingredientes aos poucos, até que se pudessem utilizar os níveis de ingredientes totais da nova formulação.

$\mathrm{Na}$ segunda etapa, os animais passaram a receber o novo sucedâneo com ajuste dos níveis de proteína bruta $(7,45 \%)$ e energia bruta $(1723,50 \mathrm{kcal} / \mathrm{kg})$, baseado na composição centesimal do leite materno de $T$. inunguis (Barbosa, 2011) (S2), por um período de 62 dias. Durante a noite, quando os animais não eram amamentados, foram fornecidos entre 3\% e 4\% do peso corporal do animal de capim-colônia (Brachiaria mutica). Os ingredientes utilizados para a formulação dos sucedâneos estão descritos na Tab. 1. 
Tabela 1. Ingredientes utilizados na elaboração dos sucedâneos do leite materno fornecidos durante os experimentos para os filhotes de peixe-boi da Amazônia (Trichechus inunguis) mantidos em cativeiro no Parque Aquático Robin C. Best/Inpa, Manaus, Amazonas

\begin{tabular}{lccc}
\hline $\begin{array}{l}\text { Ingredientes } \\
(\mathrm{g} / \mathrm{kg} \text { de leite })\end{array}$ & $\begin{array}{c}\mathrm{S} 1 \\
\text { (primeira mamada do dia) }\end{array}$ & $\mathrm{S} 1$ & $\mathrm{~S} 2$ \\
\hline Água morna & 860 & 855 & 663,6 \\
Leite bovino em pó integral & 120 & 120 & 120 \\
Óleo de canola & 20 & 20 & 69 \\
Aminomix ${ }^{\circledR 1}$ & 5 & - & 1,4 \\
Farelo de soja & - & - & 53 \\
Aveia em flocos & - & - & 53 \\
Farinha de milho & - & - & 40 \\
\hline
\end{tabular}

${ }^{1}$ Suplemento de aminoácidos, vitaminas e minerais (Vetnil, Brasil).

$\mathrm{S} 1$ = sucedâneo $1, \mathrm{~S} 2$ = sucedâneo 2 .

Foram efetuadas análises bromatológicas dos sucedâneos experimentais, seguindo-se os protocolos adotados no Laboratório de Análises Bromatológicas do Setor de Aquicultura do Inpa. No início e no fim de cada etapa experimental, foram realizadas biometrias em todos os animais e, para a avaliação do perfil metabólico, foi coletada uma amostragem sanguínea, após cada período experimental, para avaliação dos parâmetros sanguíneos.

Foi feita a análise estatística descritiva dos dados e, para avaliar a normalidade das variáveis, utilizou-se o teste de D'Agostinho. Foram aplicados o teste $\mathrm{t}$ de Student pareado $(\mathrm{P}<0,05)$ para comparação entre variáveis normais e o de Wilcoxon pareado $(\mathrm{P}<0,05)$ para as não normais. As análises estatísticas foram realizadas com o uso de software estatístico Bioestat 5.0.

\section{RESULTADOS E DISCUSSÃO}

A Tab. 2 apresenta a composição química do leite materno de peixe-boi da Amazônia (Barbosa, 2011), do capim-colônia (Brachiaria mutica), do sucedâneo 1 e do sucedâneo 2 .

Tabela 2. Composição química do leite materno de peixe-boi da Amazônia (Trichechus inunguis), da área foliar do capim-colônia (Brachiaria mutica), do sucedâneo rotineiramente fornecido (S1) e do novo sucedâneo formulado (S2), para os filhotes mantidos em cativeiro no Parque Aquático Robin C. Best/Inpa, Manaus, Amazonas

\begin{tabular}{lccccc}
\hline Nutriente & $\begin{array}{c}\text { Leite } \\
\text { materno }^{1}\end{array}$ & $\begin{array}{c}\text { Capim- } \\
\text { colônia }\end{array}$ & $\begin{array}{c}\text { S1 com } \\
\text { Aminomix }\end{array}$ & $\begin{array}{c}\text { S1 sem } \\
\text { Aminomix }\end{array}$ & Sucedâneo 2 \\
\hline Umidade (\%) & 78,06 & 36,70 & 86,25 & 86,75 & 68,24 \\
Sólidos totais (\%) & 21,87 & 63,30 & 13,75 & 13,25 & 31,76 \\
Proteína bruta (\%) & 6,75 & 14,62 & 3,40 & 3,33 & 7,45 \\
Lipídeos (\%) & 14,02 & 0,82 & 2,05 & 2,11 & 10,11 \\
Matéria mineral (\%) & 1,10 & 8,16 & 1,08 & 0,77 & 1,31 \\
Carboidratos (\%) & - & 27,68 & 7,22 & 7,04 & 12,49 \\
Fibra bruta (\%) & - & 12,02 & - & - & 0,40 \\
Energia bruta (kcal/kg) & 1531,90 & 2246,60 & 609,30 & 604,70 & 1723,50 \\
\hline
\end{tabular}

${ }^{1}$ Fonte: (Barbosa, 2011).

S1 = sucedâneo $1, \mathrm{~S} 2$ = sucedâneo 2 .

Na Tab. 3, encontram-se descritos os valores dos parâmetros sanguíneos analisados no perfil metabólico dos filhotes de peixe-boi da Amazônia
(Trichechus inunguis) alimentados com diferentes sucedâneos do leite materno. 
Tabela 3. Comparação dos valores médios ( \pm DP) para os dados bioquímicos sorológicos e hematológicos dos filhotes de peixe-boi da Amazônia (Trichechus inunguis) em cativeiro $(\mathrm{n}=10)$, alimentados com diferentes sucedâneos do leite materno no Parque Aquático Robin C. Best/Inpa, Manaus, Amazonas

\begin{tabular}{|c|c|c|c|c|}
\hline \multirow{2}{*}{ Parâmetros bioquímicos } & \multirow{2}{*}{$\begin{array}{c}\text { Tipo de } \\
\text { sucedâneo }\end{array}$} & \multicolumn{2}{|c|}{ Análise geral } & \multirow{2}{*}{$\begin{array}{c}\text { Média } \pm \mathrm{DP} \text { da } \\
\text { diferença }\end{array}$} \\
\hline & & Média \pm DP & Min - Máx & \\
\hline \multirow{2}{*}{ Proteínas totais $(\mathrm{g} / \mathrm{dL})$} & $\mathrm{S} 1^{\mathrm{b}}$ & $7,50 \pm 0,62$ & $6,20-8,30$ & $0,81 \pm 0,28$ \\
\hline & $\mathrm{S} 2^{\mathrm{c}}$ & $6,69 \pm 0,53^{*}$ & $5,80-7,50$ & $\mathrm{P}<0,0001$ \\
\hline \multirow{2}{*}{ Albumina $(\mathrm{g} / \mathrm{dL})^{\mathrm{a}}$} & $\mathrm{S} 1$ & $2,91 \pm 0,24$ & $2,40-3,10$ & \\
\hline & S2 & $2,65 \pm 0,15^{*}$ & $2,40-2,90$ & $\mathrm{P}=0,0038$ \\
\hline \multirow{2}{*}{ Globulina (g/dL) } & S1 & $4,59 \pm 0,39$ & $3,80-5,20$ & $0,55 \pm 0,23$ \\
\hline & S2 & $4,04 \pm 0,42 *$ & $3,40-4,80$ & $\mathrm{P}<0,0001$ \\
\hline \multirow{2}{*}{ Glicose (mg/dL) } & S1 & $53,50 \pm 9,28$ & $39,00-68,00$ & $16,80 \pm 14,72$ \\
\hline & S2 & $36,70 \pm 12,90 *$ & $10,00-53,00$ & $\mathrm{P}=0,0028$ \\
\hline \multirow{2}{*}{ Lipídeos totais (mg/dL) } & S1 & $733,70 \pm 128,07$ & $509,00-1007,00$ & $214,40 \pm 134,10$ \\
\hline & S2 & $519,30 \pm 44,99 *$ & $440,00-587,00$ & $\mathrm{P}=0,0003$ \\
\hline \multirow{2}{*}{ Colesterol (mg/dL) } & S1 & $307,20 \pm 84,42$ & $157,00-458,00$ & $127,10 \pm 80,81$ \\
\hline & S2 & $180,10 \pm 35,57 *$ & $118,00-247,00$ & $\mathrm{P}=0,0004$ \\
\hline \multirow{2}{*}{ Triglicerídeos $(\mathrm{mg} / \mathrm{dL})^{\mathrm{a}}$} & S1 & $153,70 \pm 59,38$ & $74,00-276,00$ & $\mathrm{P}-00025$ \\
\hline & S2 & $65,20 \pm 22,09^{*}$ & $48,00-119,00$ & $\mathrm{P}=0,0025$ \\
\hline \multirow{2}{*}{ Cálcio (mg/dL) } & S1 & $11,30 \pm 0,51$ & $10,60-12,20$ & $1,13 \pm 0,51$ \\
\hline & S2 & $10,17 \pm 0,25 *$ & $9,60-10,50$ & $\mathrm{P}<0,0001$ \\
\hline \multirow{2}{*}{ Fósforo (mg/dL) } & S1 & $6,57 \pm 0,84$ & $5,60-8,00$ & $0,62 \pm 1,04$ \\
\hline & $\mathrm{S} 2$ & $5,95 \pm 0,74^{*}$ & $4,50-7,10$ & $\mathrm{P}=0,0461$ \\
\hline \multirow{2}{*}{ Magnésio (mg/dL) } & $\mathrm{S} 1$ & $3,12 \pm 0,20$ & $2,70-3,40$ & $0,06 \pm 0,23$ \\
\hline & $\mathrm{S} 2$ & $3,06 \pm 0,34$ & $2,40-3,70$ & $\mathrm{P}=0,2172$ \\
\hline \multirow{2}{*}{ Sódio (mmol/L) } & S1 & $143,47 \pm 0,99$ & $142,00-145,00$ & $-4,69 \pm 1,20$ \\
\hline & S2 & $148,16 \pm 1,15^{*}$ & $146,70-149,80$ & $\mathrm{P}<0,0001$ \\
\hline \multirow{2}{*}{ Potássio $(\mathrm{mmol} / \mathrm{L})^{\mathrm{a}}$} & S1 & $4,15 \pm 0,22$ & $3,80-4,60$ & \\
\hline & $\mathrm{S} 2$ & $3,54 \pm 0,19 *$ & $3,30-4,00$ & $\mathrm{P}=0,0025$ \\
\hline \multirow{2}{*}{ Parâmetros hematológicos } & Tipo de & \multicolumn{2}{|c|}{ Análise geral } & Média \pm DP da \\
\hline & sucedâneo & Média \pm DP & Min - Máx & diferença \\
\hline \multirow{2}{*}{ Hematócrito (\%) } & $\mathrm{S} 1$ & $36,94 \pm 3,94$ & $32,50-44,40$ & $0,73 \pm 2,74$ \\
\hline & S2 & $36,21 \pm 1,92$ & $33,90-40,00$ & $\mathrm{P}=0,2110$ \\
\hline \multirow{2}{*}{ Hemoglobina $(\mathrm{g} / \mathrm{dL})^{\mathrm{a}}$} & S1 & $12,88 \pm 1,39$ & $11,30-15,20$ & $P=0.0104$ \\
\hline & S2 & $11,82 \pm 0,69 *$ & $10,80-12,80$ & $\mathrm{P}=0,0104$ \\
\hline
\end{tabular}

aados analisados pelo teste de Wilcoxon pareado, ${ }^{\text {b}}$ sucedâneo 1, ${ }^{\mathrm{c}}$ sucedâneo 2, DP = desvio-padrão.

*Diferença significativa.

Os valores médios de proteínas totais (PT), albumina (ALB) e globulina (GLO) no sangue dos peixes-bois alimentados com S2 apresentaram-se significativamente diminuídos quando comparados aos valores obtidos após a administração do S1. Valores proteicos sanguíneos, quando diminuídos simultaneamente, podem indicar uma maior hidratação do organismo, como sugerido por Lassen (2006).

Considerando-se que os animais que receberam os dois sucedâneos tinham o mesmo tratamento e permaneceram com a mesma rotina, os resultados sugerem que os filhotes, quando alimentados com $\mathrm{S} 2$, mantiveram-se mais hidratados. Isso favorece as reações orgânicas para seu desenvolvimento pelo fato de o S2 proporcionar, em média, maior ganho de peso $(12,3 \mathrm{~kg})$ e crescimento $(7,85 \mathrm{~cm})$ que o $\mathrm{S} 1$, que proporcionou ganho de peso de
$6,31 \mathrm{~kg}$ e crescimento de $5,37 \mathrm{~cm}$ (dados não publicados).

Os valores de proteínas totais deste estudo foram semelhantes aos já encontrados por outros pesquisadores para T. inunguis (RodriguezChacon, 2001; Carmo, 2009; Colares et al., 2011; Mello et al., 2011). Contudo, os níveis de ALB e GLO apresentaram-se abaixo e acima dos valores já encontrados, respectivamente.

Carmo (2009), ao determinar valores de referência para T. inunguis, observou níveis de PT de $6,2 \pm 1,4 \mathrm{~g} / \mathrm{dL}$ para filhotes machos e de $6,3 \pm 0,8 \mathrm{~g} / \mathrm{dL}$ para filhotes fêmeas, valores para ALB e GLO de $4,6 \pm 0,3 \mathrm{~g} / \mathrm{dL}$ e de $2,5 \pm 1,2 \mathrm{~g} / \mathrm{dL}$, respectivamente, para machos, e de $4,2 \pm 1,1 \mathrm{~g} / \mathrm{dL}$ e $2,2 \pm 0,7 \mathrm{~g} / \mathrm{dL}$, respectivamente, para fêmeas. A autora também destaca concentrações de PT, ALB 
e GLO encontradas para filhotes machos amamentados pela mãe $(\mathrm{PT}=6,6 \pm 0,4 \mathrm{~g} / \mathrm{dL}$, $\mathrm{ALB}=3,7 \pm 0,7 \mathrm{~g} / \mathrm{dL}$ e $\mathrm{GLO}=2,7 \pm 0,5 \mathrm{~g} / \mathrm{dL}$ ) e para filhotes machos alimentados com fórmula láctea artificial $(\mathrm{PT}=6,2 \pm 1,4 \mathrm{~g} / \mathrm{dL}, \mathrm{ALB}=4,6 \pm 0,3 \mathrm{~g} / \mathrm{dL}$ e $\mathrm{GLO}=2,5 \pm 1,2 \mathrm{~g} / \mathrm{dL})$.

Colares et al. (2011), ao realizarem trabalhos com 10 peixes-bois da Amazônia cativos (cinco machos e cinco fêmeas), observaram valores médios ( \pm desvio-padrão) de PT de $6,3 \pm 0,4 \mathrm{~g} / \mathrm{dL}$, ALB de 3,5 $\pm 0,2 \mathrm{~g} / \mathrm{dL}$ e GLO de 2,8 $\pm 0,4 \mathrm{~g} / \mathrm{dL}$.

Rodriguez-Chacon (2001) trabalhou com cinco filhotes de peixe-boi da Amazônia, entre machos e fêmeas, amamentados com sucedâneo produzido de leite bovino em pó integral, manteiga sem sal, melão, gema de ovo cru e suplemento vitamínico-mineral, obtendo valores de $\mathrm{PT}=6,3 \pm 1,2 \mathrm{~g} / \mathrm{dL}$ durante o período de amamentação, $7,8 \pm 2,9 \mathrm{~g} / \mathrm{dL}$ no período de restrição láctea e 9,5 $\pm 2,6 \mathrm{~g} / \mathrm{dL}$ após o desmame. Os dados da autora indicaram aumento de PT provavelmente pela menor ingestão de líquido durante a fase de restrição e desmame, o que ocasionou menor hidratação orgânica e concentração de metabólitos.

Mello et al. (2011) avaliaram os parâmetros bioquímicos de peixes-bois da Amazônia cativos, incluindo filhotes, subadultos e adultos, e encontraram valores médios de $6,9 \pm 0,5 \mathrm{~g} / \mathrm{dL}$ para PT e de $3,4 \pm 0,4 \mathrm{~g} / \mathrm{dL}$ para ALB.

A albumina é a proteína mais abundante no sangue, representando de $35 \%$ a $50 \%$ da proteína plasmática total (Hendrix, 2006). A variação na concentração de albumina no sangue, quando acompanhada de valores normais de globulina, pode ser um indicador útil de deficiência dietética mais prolongada em proteínas, pois a síntese de albumina ocorre no fígado a partir de aminoácidos essenciais (Lassen, 2006; Contreras, 2000). Os níveis mais baixos de albumina observados no sangue dos animais deste estudo, quando alimentados com o $\mathrm{S} 2$, poderiam sugerir um aporte insuficiente de aminoácidos essenciais na dieta, independente do teor de proteína bruta, ou ainda a ocorrência de parasitismo intestinal, que dificulta a absorção de aminoácidos e provoca perdas de sangue pela alimentação dos parasitos. Contudo, os dados deste estudo podem refletir uma condição normal do perfil metabólico proteico, tendo em vista que o S2 foi formulado com base nos níveis médios de nutrientes que compõem o leite materno de $T$. inunguis e que os animais, quando amamentados com $\mathrm{S} 2$, obtiveram maior ganho de peso e crescimento.

Valores de globulinas mais elevados que de albumina para potros em fase de crescimento foram relacionados ao desenvolvimento imunológico do animal (Mundim, 2008). Os níveis mais elevados de globulinas observados nos animais do presente estudo também podem estar relacionados ao seu desenvolvimento imunológico, tendo em vista que os peixes-bois também estão expostos a antígenos ambientais.

$\mathrm{Na}$ presente pesquisa, houve uma redução significativa $(\mathrm{P}=0,0028)$ nas concentrações sorológicas de glicose quando os animais foram alimentados com S2 (36,70 $\pm 12,90 \mathrm{mg} / \mathrm{dL}) \mathrm{em}$ relação à amamentação com S1 $(53,50 \pm 9,28 \mathrm{mg} / \mathrm{dL})$.

Colares et al. (2011) obtiveram valor médio de glicose de $43,3 \pm 7,50 \mathrm{mg} / \mathrm{dL}$ para $\mathrm{T}$. inunguis Formatado: Cor da fonte: Roxo 6 alimentados com Brachiaria mutica e observaram que os níveis glicêmicos obtidos no estudo foram semelhantes aos níveis glicêmicos de ruminantes (bovinos e ovinos) e menores que de mamíferos herbívoros não ruminantes (cavalo). Para tais autores, essa tendência a valores glicêmicos baixos, está relacionada ao baixo metabolismo que o peixe-boi da Amazônia apresenta, o qual necessita de menos glicose circulante para suprir suas necessidades metabólicas.

Para elefantes-asiáticos (Elephas maximus), os níveis glicêmicos também são baixos, e isso pode ser explicado pelo fato de esse animal utilizar os ácidos graxos voláteis como fonte energética primária em vez de glicose, assemelhando-se ao metabolismo dos ruminantes (Nirmalan e Nair, 1969). Situação semelhante poderia acontecer com peixe-boi da Amazônia, uma vez que os sirênios produzem ácidos graxos voláteis no ceco a partir da degradação de fibras vegetais e esses compostos podem fazer parte da receita energética desses animais (Burn e Odell, 1987).

Reece (2006) relatou valores pré-prandiais de glicose para bezerros lactentes que variaram entre 80 e $120 \mathrm{mg} / \mathrm{dL}$ e, para bovinos adultos, entre $40 \mathrm{e}$ $80 \mathrm{mg} / \mathrm{dL}$. A redução dos valores médios de glicose em função da idade é um reflexo do 
desenvolvimento do rúmen e da adaptação do metabolismo dos bezerros, que iniciam a utilização dos produtos provenientes da fermentação ruminal. Dessa forma, a glicose plasmática passa a ser proveniente principalmente da gliconeogênese hepática (Costa et al., 2007).

Os níveis de glicose sanguínea de $T$. inunguis possuem uma tendência a diminuir com o avanço da idade, mantendo-se baixos durante a idade adulta (Carmo, 2009; Colares et al., 2011; Mello et al., 2011). Os níveis de glicose, no entanto, diminuíram com a amamentação com o S2, que tinha em sua composição maior teor de fibra bruta, quando comparado com o $\mathrm{S} 1$, que não apresentava fibra bruta em sua composição (Tab. 2). Considerando-se que os peixes-bois são exclusivamente herbívoros e monogástricos e que realizam digestão fermentativa no intestino grosso, semelhantemente ao cavalo (Burn, 1986), sugere-se, neste trabalho, que o peixe-boi da Amazônia direciona seu metabolismo energético para utilização primária de ácidos graxos voláteis à medida que sua dieta passa a ter composição maior em fibras, consolidando-se na fase adulta, quando passa a se alimentar apenas de vegetais.

Os valores médios de LT, COL e TRI no sangue dos animais deste estudo ao final da amamentação com S2 foram bem inferiores aos valores encontrados quando os animais foram alimentados com S1. Como as coletas de sangue foram realizadas em torno de 14 horas após restrição láctea, supõe-se que a concentração mais elevada de lipídeos sanguíneos observada nos animais amamentados com S1 não seja pósprandial, mas que tenha ocorrido devido ao balanço energético negativo e à maior mobilização de reservas energéticas, pelo fato de o S1 ter um aporte bem menor em energia quando comparado com o S2. Pode-se observar também que o S2 possuía maior concentração de carboidratos, o que poderia levar à maior secreção e ação de insulina, reduzindo a lipólise e diminuindo os níveis de triglicerídeos nos animais, quando amamentados com S2 (Lansen e Fettman, 2006).

Comparando-se o perfil lipídico dos animais deste estudo com o obtido por Rodriguez-Chacon (2001), as concentrações de colesterol e triglicerídeos, quando os animais foram alimentados com $\mathrm{S} 1$, foram semelhantes às encontradas para filhotes em amamentação com fórmula láctea artificial produzida pela autora a partir de leite bovino em pó integral, manteiga sem sal, melão, gema de ovo cru e suplemento vitamínico-mineral. Quando amamentados com S2, os animais apresentaram valores de colesterol semelhantes aos valores de filhotes em fase de restrição e de triglicerídeos semelhantes aos animais desmamados.

Carmo (2009) obteve valores do perfil lipídico para filhotes de $T$. inunguis amamentados com leite materno (LT $=1161,0 \pm 373,0 \mathrm{mg} / \mathrm{dL}$, $\mathrm{COL}=356,7 \pm 56,0 \mathrm{mg} / \mathrm{dL} \quad$ e $\quad \mathrm{TRI}=171,0 \pm 29,4 \mathrm{mg} / \mathrm{dL}$ ) e para filhotes amamentados com formulação láctea artificial ( $\mathrm{LT}=544,4 \pm 69,5 \mathrm{mg} / \mathrm{dL}$, $\mathrm{COL}=208,0 \pm 36,6 \mathrm{mg} / \mathrm{dL}$ e $\mathrm{TRI}=93,1 \pm 28,4 \mathrm{mg} / \mathrm{dL}$ ). Segundo a autora, os baixos níveis de colesterol e triglicerídeos nos filhotes amamentados artificialmente podem ter ocorrido devido ao fato de a composição dos lipídeos utilizados nas formulações artificiais (origem vegetal) ser diferente da composição dos lipídeos do leite materno. Assim, essas fontes lipídicas de origem vegetal não são absorvidas pelo organismo dos animais com a mesma eficácia de absorção da gordura do leite materno (Nelson e Cox, 2005).

Neste estudo, os valores de lipídeos totais, colesterol e triglicerídeos também foram inferiores aos encontrados por Carmo (2009) para filhotes amamentados pela mãe. É possível que, além da diferença de gordura da dieta láctea entre os peixes-bois que consomem fórmula láctea artificial e os que mamam na mãe, o metabolismo energético possa ser influenciado pelo nível de estresse do animal, pelo horário de coleta sanguínea e pelo jejum prolongado. Animais alimentados artificialmente são mantidos em piscinas menores e na companhia de, no máximo, mais um animal da mesma faixa etária. Os filhotes mantidos com a mãe ficam em tanques maiores, muitas vezes com outros animais adultos, e realizam maior esforço físico para natação, podendo mobilizar maior reserva energética. Em condições de estresse por esforço físico, pode-se esperar um aumento na concentração de triglicerídeos devido ao bloqueio na ação da insulina e do efeito hiperglicemiante gerado pelas catecolaminas e pelo cortisol circulantes. Com isso, ocorre um balanço energético negativo, semelhante ao que ocorre quando um equino é submetido a jejum alimentar, havendo lipólise e mobilização de outras fontes energéticas (Dugat et al., 2010). 
Quanto ao metabolismo mineral, os valores séricos estiveram dentro dos padrões normais estabelecidos para T. inunguis (Carmo, 2009). A leve diminuição de $\mathrm{Ca}, \mathrm{P}$ e K com a utilização do $\mathrm{S} 2$ pode estar relacionada à maior demanda desses minerais para o crescimento (McDowell, 1999), considerando que os animais amamentados com $\mathrm{S} 2$ cresceram e ganharam peso mais rápido do que quando amamentados com S1.

Os níveis aumentados de $\mathrm{Na}$ no sangue dos animais amamentados com S2 podem estar refletindo o incremento desse mineral na dieta, considerando que os ingredientes utilizados na elaboração do sucedâneo 2 podem fornecer quantidades maiores desse mineral do que os ingredientes do sucedâneo 1 .

Para os parâmetros hematológicos, não houve alterações significativas para $\mathrm{Ht}$ entre as amostras coletadas dos animais amamentados com S1 e S2 $(\mathrm{P}=0,2110)$, e os animais, quando amamentados com S2, apresentaram diminuição nos valores de $\mathrm{Hb}$ em relação a quando foram amamentados com $\mathrm{S} 1$. Os valores de $\mathrm{Ht}$ e $\mathrm{Hb}$ observados foram considerados normais para a espécie (Carmo, 2009).

\section{CONCLUSÕES}

Os dados deste trabalho fornecem bases para novos estudos que visem elucidar as importantes vias metabólicas dos peixes-bois da Amazônia na utilização da proteína e da energia da dieta, bem como os mecanismos de regulação energética endógena. Este estudo também pode servir como base para novos trabalhos na busca da determinação das exigências nutricionais para a espécie.

Nenhuma das dietas lácteas oferecidas aos filhotes de peixe-boi proporcionou valores bioquímicos séricos que sugerissem alguma patologia. Os valores obtidos mantiveram-se dentro dos padrões já observados para a espécie, com exceção da albumina e da globulina, que tiveram valores diminuídos e aumentados quando comparados aos valores já encontrados para a espécie, respectivamente. O perfil metabólico realizado com filhotes de peixes-bois da Amazônia, mantidos em cativeiro, mostrou ser uma ferramenta viável e de grande importância no auxílio da avaliação nutricional desses animais.

\section{AGRADECIMENTOS}

Ao médico veterinário Anselmo D’Affonseca, do Inpa, e à equipe Prevet; à Fundação de Hematologia e Hematoterapia do Estado do Amazonas (Hemoam); à Fundação de Amparo à Pesquisa do Estado do Amazonas (Fapeam); à Associação Amigos do Peixe-boi (Ampa); e à Petrobrás Ambiental, pelo apoio financeiro do estudo.

\section{REFERÊNCIAS}

AMARAL, R.S.; SILVA, V.M.; ROSAS, F.C. Body weight/length relationship and mass estimation using morphometric measurements in Amazonian manatees Trichechus inunguis (Mammalia: Sirenia). Mar. Biodiversity Rec., v.3, p.105, 2010.

BARBOSA, P.S. Composição química do leite do peixe-boi da Amazônia (Trichechus inunguis Natterer, 1883) em cativeiro nos diferentes estágios de lactação. 2011. 50f. Dissertação (Mestrado em Biologia de Água Doce e Pesca Interior) - Instituto Nacional de Pesquisas da Amazônia, Manaus, AM.

BEST R.C.; RIBEIRO G.A.; YAMAKOSHI M.; SILVA V.M.F. Artificial feeding for unwearied Amazonian manatees. Int. Zoo Yearbook, v.22, p.263267,1982

BURN D.M. The digestive strategy and efficiency of the West Indian manatee, Trichechus manatus. Comp. Biochemi. Physiol. Part A Physiol., v.85, p.139-142, 1986.

BURN, D.M.; ODELL, D.K. Volatile fatty acid concentrations in the digestive tract of the west indian manatee, (Trichechus manatus). Comp. Biochem. Physiol. Part B Comp. Biochem., v.88, p.47-49, 1987.

CARMO, T.L.L do. Hematologia e bioquímica sanguínea do peixe-boi da Amazônia Trichechus inunguis (Natterer, 1883). 2009. 50f. Dissertação (Mestrado em Biologia de Água Doce e Pesca Interior) - Instituto Nacional de Pesquisas da Amazônia, Manaus, AM.

COLARES E.P.; MOREIRA G.R.; RIBEIRO G.A. Amamentação do peixe-boi amazônico (Trichechus inunguis) em cativeiro. REUNIÃO DE TRABALHO DE ESPECIALISTAS EM MAMÍFEROS AQUÁTICOS DA AMERICA DO SUL, 2., 1987, Rio de Janeiro. Anais... Rio de Janeiro: FBCN, 1987. p.4041.

COLARES, E.P.; COLARES, I.G.; DO AMARAL, A.D. Parâmetros bioquímicos do sangue do peixe-boi da Amazônia (Trichechus inunguis, Mammalia: Sirenia). Nat. Res., v.1, p.15-20, 2011. 
CONTRERAS, P. Indicadores do metabolismo proteico utilizados nos perfis metabólicos de rebanhos. In: GONZALEZ, F.H.D.; BARCELLOS, J.O.; OSPINA, H.; RIBEIRO, L.A.O. Perfil metabólico em ruminantes: seu uso em nutrição e doenças nutricionais. Porto alegre: Universidade Federal do Rio Grande do Sul, 2000. p.23-30.

COSTA, T.C.; COELHO, S.G.; FONTES, F.A.P.V. et al. Consumo de mistura mineral e desempenho de bezerros alimentados com sucedâneos do leite. Arq. Bras. Med. Vet. Zootec., v.59, p.749-758, 2007.

D'AFFONSECA NETO, J.A.; VERGARAPARENTE, J.E. Sirênia (Peixe-boi-da-Amazônia, Peixe-boi-marinho). In: CUBAS, Z.S.; SILVA, J.C.R.; CATÃO-DIAS, J.L. (Eds). Tratado de animais selvagens - medicina veterinária. São Paulo: Rocca, 2006. p.701-714.

DUGAT, S.L.; TAYLOR, T.S.; MATTHEWS, N.S.; GOLD, J.R. Values for triglycerides, insulin, cortisol and ACTH in a herd of normal donkeys. J. Equine Vet. Sci., v.30, p.141-144, 2010.

FERREIRA A.M.; TORRES C.A.A. Glicose e lipídeos totais como indicadores de "status" nutricional de bovinos. Rev. Soc. Bras. Zootec., v.21, p.339-345, 1992.

GONZÁLEZ, F.H.D.; BARCELLOS, J.O.; OSPINA, H.; RIBEIRO, L.A.O. Perfil metabólico em ruminantes: seu uso em nutrição e doenças nutricionais. Porto alegre: Universidade Federal do Rio Grande do Sul, 2000. p.23-30.

GUTERRES-PAZIN， M.G.; MARMONTEL， M.; ROSAS, F.C.; PAZIN, V.F.; VENTICINQUE, E.M. Feeding Ecology of the Amazonian Manatee (Trichechus inunguis) in the Mamirauá and Amanã Sustainable Development Reserves, Brazil. Aquat. Mamm., v.40, p.139-149, 2014.

HENDRIX, C.M. Procedimentos laboratoriais para técnicos veterinários. São Paulo: Roca, 2006. 556p.

LASSEN, E.D. Avaliação laboratorial das proteínas do plasma e do soro sanguíneo. In: THRALL, M.A.; BAKER, D.C.; CAMPBELL, T.W. et al. Hematologia e bioquímica clínica veterinária. São Paulo: Roca, 2006. p.376-390.

LASSEN, E.D.; FETTMAN, M.J. Avaliação laboratorial dos lipídeos. In: THRALL, M.A.; BAKER, D.C.; CAMPBELL, T.W. et al. Hematologia $e$ bioquímica clínica veterinária. São Paulo: Roca, 2006. p.394-402.

McDOWELL, L.R. Minerais para ruminantes sob pastejo em regiões tropicais, enfatizando o Brasil. Gainesville: University of Florida, 1999. 92p.
MELLO, D.M.D.; SILVA, V.; ROSAS, F. Serum biochemical analytes in captive Amazonian manatees (Trichechus inunguis). Vet. Clinic. Pathol., v.40, p.7477, 2011.

MUNDIM, A.V. Perfil bioquímico sérico em potros bretão postier e cães doberman em fase de crescimento e de cabras saanen nos diferentes estágios de lactação. 2008. 76f. Tese (Doutorado em Genética e Bioquímica) - Universidade Federal de Uberlândia, Uberlândia,MG.

NELSON, D.L.; COX, M.M. Principles of biochemistry. New York: W.H. Freeman and Company, 2005. 1120p.

NIRMALAN, G.; NAIR, S.G. Biochemical studies on the blood of Indian elephants (Elephas maximus). Res. Vet. Sci., v.10, p.176-180, 1969.

OLIVEIRA, R.P.M.; MADURO, A.H.P.; OLIVEIRA, F.F.; LIMA, E.S. Perfil metabólico de ovelhas Santa Inês em diferentes fases de gestação criadas em sistema semi-intensivo no estado do Amazonas. Ciênc. Anim. Bras., v.15, p.81-86, 2014.

PAYNE, J.M.; DEW, S.M.; MANSTON, R. et al. The use of metabolic profile test in dairy herds. Vet. Rec., v.87, p.150-158, 1970.

REECE, W.O. Fisiologia dos animais domésticos. 12.ed. Rio de Janeiro: Guanabara Koogan, 2006. 856p.

RIBEIRO, L.A.O.; GONZÁLEZ, F.H.D.; CONCEIÇÃO, T.R. et al. Perfil metabólico de borregas Corriedale em pastagem nativa do Rio Grande do Sul. Acta Sci. Vet., v.31, p.167-170, 2003.

RODRIGUEZ-CHACÓN, Z. M. Características alimentares e nutricionais do peixe-boi da Amazônia Trichechus inunguis (Mammalia, Sirenia), em condição de cativeiro. 2001. 170f. Tese (Doutorado em Biologia de Água Doce e Pesca Interior) - Instituto Nacional de Pesquisas da Amazônia, Manaus, AM.

ROSAS, F.C.W. Crescimento de filhotes de peixe-boi da Amazônia (Trichechus inunguis) criados com leite artificial. REUNIÃO DE ESPECIALISTAS EM MAMÍFEROS AQUÁTICOS DA AMERICA DO SUL, 5., 1992. Anais... [s.1.]: [s.n.], 1992. v.59.

SILVA, F.M.O. Perfil hematológico, bioquímico sérico, nutricional e biométrico de filhotes de peixe-boi marinho (Trichechus manatus manatus, Linnaeus, 1758) mantidos em cativeiro no centro mamíferos aquáticos - CMA/ICMBio, Ilha de Itamaracá, PE. 2008. 83f. Dissertação (Mestrado em Ciência Veterinária) - Universidade Federal de Pernambuco, Recife, PE. 